\title{
Article
}

\section{THE FATE OF SUPERMASSIVE BLACK HOLES AND THE EVOLUTION OF THE MBH- j RELATION IN MERGING GALAXIES: THE EFFECT OF GASEOUS DISSIPATION}

Kazantzidis, Stelios, Mayer, Lucio, Colpi, Monica, Madau, Piero, Debattista, Victor P, Wadsley, James, Stadel, Joachim, Quinn, Thomas and Moore, Ben

Available at https://clok.uclan.ac.uk/16884/

Kazantzidis, Stelios, Mayer, Lucio, Colpi, Monica, Madau, Piero, Debattista, Victor P orcid iconORCID: 0000-0001-7902-0116, Wadsley, James, Stadel, Joachim, Quinn, Thomas and Moore, Ben (2005) THE FATE OF SUPERMASSIVE BLACK HOLES AND THE EVOLUTION OF THE MBH-j RELATION IN MERGING GALAXIES: THE EFFECT OF GASEOUS DISSIPATION. The Astrophysical Journal Letters, 63 (2). L67-L70. ISSN 2041-8205

It is advisable to refer to the publisher's version if you intend to cite from the work. http://dx.doi.org/10.1086/430139

For more information about UCLan's research in this area go to http://www.uclan.ac.uk/researchgroups/ and search for <name of research Group>.

For information about Research generally at UCLan please go to http://www.uclan.ac.uk/research/

All outputs in CLoK are protected by Intellectual Property Rights law, including Copyright law. Copyright, IPR and Moral Rights for the works on this site are retained by the individual authors and/or other copyright owners. Terms and conditions for use of this material are defined in the policies page. 


\title{
THE FATE OF SUPERMASSIVE BLACK HOLES AND THE EVOLUTION OF THE $M_{\mathrm{BH}^{-}} \sigma$ RELATION IN MERGING GALAXIES: THE EFFECT OF GASEOUS DISSIPATION
}

\author{
Stelios Kazantzidis, ${ }^{1}$ Lucio Mayer, ${ }^{1}$ Monica Colpi,${ }^{2}$ Piero Madau, ${ }^{3}$ Victor P. Debattista, ${ }^{4}$ \\ James Wadsley, Joachim Stadel, ${ }^{1}$ Thomas Quinn, ${ }^{6}$ and Ben Moore ${ }^{1}$ \\ Received 2004 July 20; accepted 2005 March 10; published 2005 March 22
}

\begin{abstract}
We analyze the effect of dissipation on the orbital evolution of supermassive black holes (SMBHs) using highresolution self-consistent gasdynamical simulations of binary equal- and unequal-mass mergers of disk galaxies. The galaxy models are consistent with the $\Lambda \mathrm{CDM}$ paradigm of structure formation, and the simulations include the effects of radiative cooling and star formation. We find that equal-mass mergers always lead to the formation of a close SMBH pair at the center of the remnant, with separations limited solely by the adopted force resolution of $\sim 100 \mathrm{pc}$. Instead, the final SMBH separation in unequal-mass mergers depends sensitively on how the central structure of the merging galaxies is modified by dissipation. In the absence of dissipation, the satellite galaxy can be entirely disrupted before the merger is completed, leaving its SMBH wandering at a distance too far from the center of the remnant for the formation of a close pair. In contrast, gas cooling facilitates the pairing process by increasing the resilience of the companion galaxy to tidal disruption. Moreover, we demonstrate that merging disk galaxies constructed to obey the $M_{\mathrm{BH}^{-}} \sigma$ relation move relative to it depending on whether they undergo a dissipational or collisionless merger, regardless of the mass ratio of the merging systems. Collisionless simulations reveal that remnants tend to move away from the mean relation, highlighting the role of gas-poor mergers as a possible source of scatter. In dissipational mergers, the interplay between strong gas inflows associated with the formation of massive nuclear disks and the consumption of gas by star formation provides the necessary fuel to the SMBHs and allows the merger remnants to satisfy the relation
\end{abstract}

Subject headings: black hole physics — cosmology: theory — galaxies: interactions — hydrodynamics — methods: numerical

\section{INTRODUCTION}

Irrefutable dynamical evidence indicates that supermassive black holes (SMBHs) with masses ranging from $10^{6}$ to above $10^{9} M_{\odot}$ reside at the centers of most galaxies hosting spheroids (Kormendy \& Richstone 1995). The available data show the existence of a remarkably tight correlation between the mass of the SMBHs, $M_{\mathrm{BH}}$, and the stellar velocity dispersion of the host galaxy spheroidal component, $\sigma$ (Ferrarese \& Merritt 2000; Gebhardt et al. 2000), suggesting a fundamental mechanism that connects SMBH assembly and galaxy formation (Silk \& Rees 1998). According to the currently favored cold dark matter (CDM) cosmological model, structures in the universe are the end result of a complex hierarchy of mergers and accretion of smaller subunits. Thus, the hierarchical buildup of SMBHs by massive seed black holes present at the center of protogalaxies and the formation of SMBH binaries appear as a natural consequence in any "bottom-up" cosmogony. Recently, the discovery of a binary active galactic nucleus (AGN) in the interacting system NGC 6240 by Komossa et al. (2003) has lent support to this picture.

The formation and subsequent orbital evolution of an SMBH pair depends on how efficiently the host galactic cores lose

\footnotetext{
${ }^{1}$ Institute for Theoretical Physics, University of Zürich, Winterthurerstrasse 190, CH-8057 Zürich, Switzerland; stelios@physik.unizh.ch.

${ }^{2}$ Dipartimento di Fisica G. Occhialini, Università di Milano Bicocca, Piazza della Scienza 3, I-20126 Milan, Italy.

${ }^{3}$ Department of Physics, University of California at Santa Cruz, 1156 High Street, Santa Cruz, CA 95064.

${ }^{4}$ Institut für Astronomie, ETH Zürich, Scheuchzerstrasse 7, CH-8093 Zürich, Switzerland.

${ }^{5}$ Department of Physics and Astronomy, McMaster University, 1280 Main Street West, Hamilton, ON L8S 4M1, Canada.

${ }^{6}$ Department of Astronomy, University of Washington, Physics/Astronomy Building, Stevens Way, Box 351580, Seattle, WA 98195.
}

angular momentum by dynamical friction during the merger. Once the two cores have merged, the SMBHs may decay further and eventually form a binary owing to the drag exerted by the background mass distribution. This problem has been investigated numerically by a number of authors (e.g., Governato et al. 1994; Makino \& Ebisuzaki 1996; Milosavljević \& Merritt 2001), but the galaxy models used in these studies were idealized, spherical systems that could at most faithfully represent the central spheroidal components of real galaxies. Hence, the larger scale dynamical evolution of the merging systems and the cosmological framework were both missing. Moreover, with the notable exception of Escala et al. (2004a, 2004b), these studies did not explore the role of a dissipative component in driving the evolution of an SMBH pair. However, if and how the initial conditions of Escala et al. are related to the larger scale dynamics involved in galaxy merging is still unclear.

In this Letter, we report on the effects of gaseous dissipation on the fate of SMBHs using high-resolution binary disk galaxy merger simulations. Governato et al. (1994) have already highlighted the difficulty of forming a close SMBH pair when tidal disruption of one of the host systems intervenes. As we illustrate below, gaseous dissipation is vital for the survival of the host galaxies by deepening their potential wells and provides the necessary fuel for the SMBH growth. Finally, we analyze the stellar kinematics and distribution of gas in the central regions of the merger remnants to investigate for the first time how merging galaxies move with respect to their initial location on the $M_{\mathrm{BH}^{-}} \sigma$ plane.

\section{NUMERICAL SIMULATIONS}

We perform four types of high-resolution simulations of binary disk galaxy mergers with mass ratios of $1: 1$ and $4: 1$ including different physical processes. We simulate purely col- 
lisionless mergers and mergers in which we follow the gas dynamics "adiabatically," i.e., without radiative cooling. A third set of simulations includes radiative cooling, while in the fourth set we also allow the cold gas to form stars. The simulations were performed with GASOLINE, a multistepping, parallel treeSPH $N$-body code (Wadsley et al. 2004). We include radiative and Compton cooling for a primordial mixture of hydrogen and helium. The star formation algorithm is based on that by Katz (1992) and reproduces the Schmidt law. The star formation efficiency was set to 0.1 , which yields a star formation rate of $1-2 M_{\odot} \mathrm{yr}^{-1}$ for models in isolation that have a disk gas mass and surface density comparable to those of the Milky Way.

The multicomponent galaxy models are constructed using the technique described by Hernquist (1993), and their structural parameters are consistent with the $\Lambda \mathrm{CDM}$ paradigm for structure formation (Mo et al. 1998). To this end, each galaxy consists of a spherical and isotropic Navarro et al. (1996) dark matter (DM) halo (Kazantzidis et al. 2004b), an exponential disk, and a spherical, Hernquist (1990) nonrotating bulge. For the basic galaxy model, we adopted parameters from the Milky Way model A1 of Klypin et al. (2002). Specifically, the DM halo had a virial mass of $M_{\text {vir }}=10^{12} M_{\odot}$, a concentration parameter of $c=12$, and a dimensionless spin parameter of $\lambda=0.031$. The mass, thickness, and resulting scale length of the disk were $M_{d}=0.04 M_{\mathrm{vir}}, z_{0}=0.1 R_{d}$, and $R_{d}=3.5 \mathrm{kpc}$, respectively. The bulge mass and scale radius were $M_{b}=$ $0.008 M_{\text {vir }}$ and $a=0.2 R_{d}$, respectively. The DM halo was adiabatically contracted to respond to the growth of the disk and bulge (Blumenthal et al. 1986), resulting in galaxy models with a central total density slope close to isothermal (Fig. 1). The companion galaxy is either a replica of the same model in equalmass mergers or a system containing one-fourth of the mass in each component in which lengths and velocities are renormalized according to the cosmological scaling for virialized systems (Mo et al. 1998). The disk scale length of the satellite galaxy is equal to $R_{d}=2.2 \mathrm{kpc}$. Given the uncertainties in the adopted $M / L$, our galaxies are consistent with the stellar mass Tully-Fisher and size-mass relations. To each of the galaxy models we added a particle representing an SMBH at the center of the bulge component that was initially at rest. For the larger galaxy model, we used an SMBH mass equal to $M_{\mathrm{BH}}=3 \times$ $10^{6} M_{\odot}$.

We considered two values for the gas fraction, $f_{g}$, namely, $10 \%$ and $50 \%$ of the total disk mass. We shut off radiative cooling at temperatures below $2 \times 10^{4} \mathrm{~K}$, which is about a factor of 2 higher than the temperature at which atomic radiative cooling would drop sharply owing to the adopted cooling function. With this choice, we take into account nonthermal pressures and approximate the simulated gas with the warm interstellar medium of a real galaxy (Barnes 2002). Moreover, this choice enables us to simulate very gas-rich disks that would otherwise become strongly gravitationally unstable and undergo widespread fragmentation. Two main encounter geometries were adopted: prograde coplanar mergers and mergers in which one of the disks was inclined with respect to the orbital plane. We also simulated a single retrograde equal-mass merger in which both disk spin vectors were antiparallel to the orbital angular momentum vector. The galaxies approached each other on parabolic orbits with pericentric distances that were $20 \%$ of the more massive galaxy's virial radius, typical of cosmological mergers. The initial separation of the halo centers was twice their virial radii, and their initial relative velocity was determined from the corresponding Keplerian or-

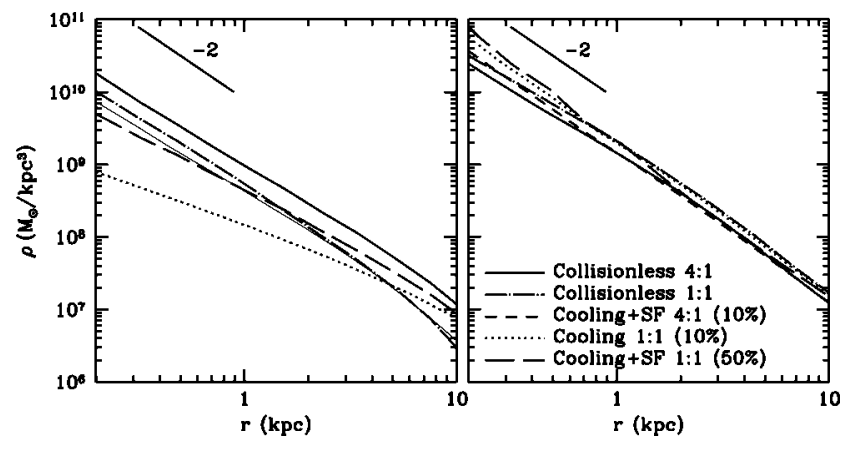

FIG. 1.-Spherically averaged density distribution for different components in the initial models (left) and some of the remnants (right) plotted from the force resolution outward. Left: Different thick curves show the total (solid curve), DM density after the adiabatic contraction due to baryons (dashed curve), and total density of baryons (dot-dashed curve) in the basic galaxy model. The dotted curve shows the DM density before the baryonic contraction. The thin solid curve shows the total density of the satellite galaxy. After the adiabatic contraction, the central density slope is close to isothermal with $\rho(r) \propto r^{-2}$ for both galaxy models. Right: Total density profiles for some of the merger remnants in the coplanar encounter geometry. The numbers in parentheses indicate the values for the gas fraction, $f_{g}$, used in the particular simulation. The center of each remnant was defined as the location of the minimum of the gravitational potential. In dissipative simulations, the remnants exhibit an increase in the central density resulting from strong gas inflows and star formation associated with the merger.

bit of two point masses. In collisionless mergers, we used $N=1.2 \times 10^{6}$ particles in total, with each galaxy consisting of $10^{5}$ disk particles, $10^{5}$ bulge particles, and $10^{6} \mathrm{DM}$ particles. The gas component in gasdynamical simulations was represented by $10^{5}$ particles. We adopted a gravitational softening of $\epsilon=0.1 \mathrm{kpc}$ for both the DM and baryonic particles of the larger galaxy, and for its $\mathrm{SMBH} \epsilon=0.03 \mathrm{kpc}$, which is small enough to follow its orbital evolution. For the satellite galaxy, all softening lengths were scaled according to $\epsilon \propto m^{1 / 3}$. In all simulations, the merger remnants were allowed to settle into equilibrium $\sim 15$ dynamical times at the disk half-mass radius after the merger was completed.

\section{RESULTS}

\subsection{SMBH Pairing in Binary Disk Galaxy Mergers}

The galaxies merge in three to five orbits (between 5.5 and 7 Gyr), depending on the mass of the companion, a timescale that is set by dynamical friction and tidal stripping (e.g., Taffoni et al. 2003). These timescales are longer than those reported in earlier studies (e.g., Barnes \& Hernquist 1996) owing to the larger and more realistic pericentric distances adopted here. In equal-mass mergers, the cuspy potentials of both galaxies are deep enough to allow the survival of their inner regions until orbital decay by dynamical friction is complete. This result holds for both collisionless and gasdynamical mergers. In the latter, during the first two orbits a strong spiral pattern appears in both the stellar and the gaseous component and mild nonaxisymmetric torques redistribute mass and angular momentum driving approximately $20 \%$ of the gas toward the center. However, the central bulges stabilize the galaxies against bar formation. Shortly before the galaxies merge, a second much stronger gas inflow occurs caused by strong tidal torquing as the galaxies perform a close flyby. However, only in the simulations with radiative cooling does this inflow result in a significant central concentration of cold $\left(T \sim 2 \times 10^{4} \mathrm{~K}\right)$ gas. In this case, more than $80 \%$ of the gas that was originally in the disks is collected within the central $500 \mathrm{pc}$. This results in a considerable steepening of the 


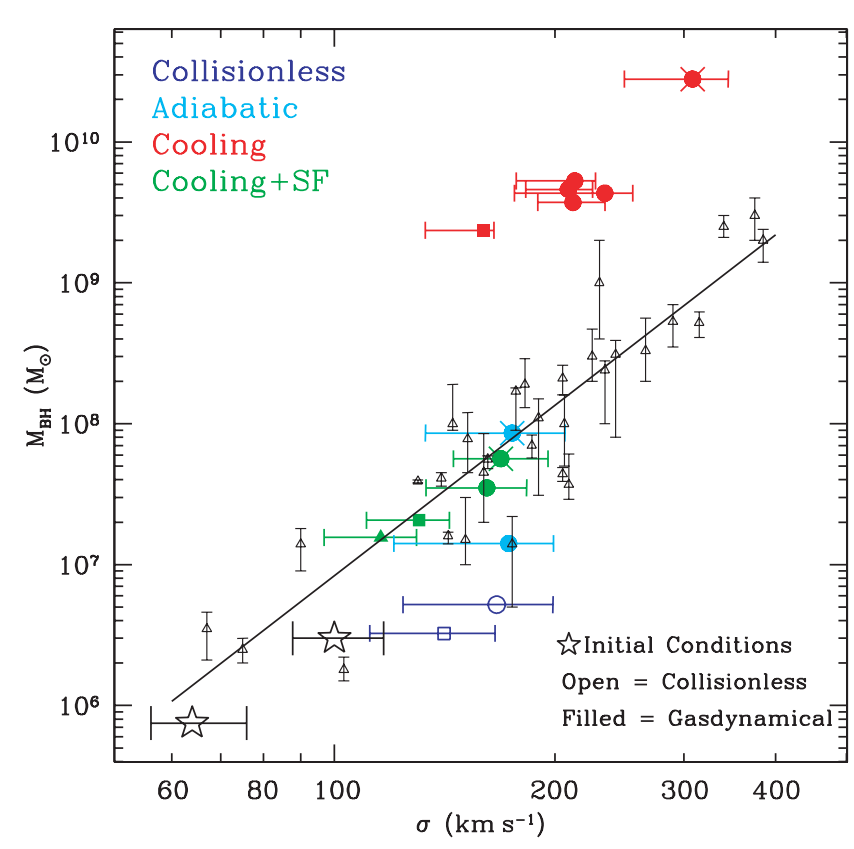

FIG. 2. $-M_{\mathrm{BH}^{-}} \sigma$ relation in binary disk galaxy mergers. The open triangles show data from the galaxy sample compiled by Tremaine et al. (2002), and the solid line corresponds to their best-fit correlation. Results for simulated remnants are shown in color, and the initial galaxy models are denoted by star symbols. Open and filled symbols correspond to collisionless and gasdynamical simulations, respectively. Circles and squares show equal- and unequal-mass mergers, respectively. The filled triangle corresponds to an unequal-mass merger in which the larger disk was inclined by $45^{\circ}$ with respect to the orbital plane. Symbols outlined with crosses correspond to mergers with $50 \%$ gas fraction in the disks. The error bars show the spread about the mean value of $\sigma$ in each remnant.

central density slope of the combined distribution of DM and baryons (Fig. 1). In adiabatic simulations, only $20 \%-30 \%$ of the initial gas mass collects within the central kiloparsec. This gas is heated to temperatures $T>10^{5} \mathrm{~K}$ by shocks and compressions during the merger and subsequently expands since it cannot radiate away its energy. Large central gas inflows were already noticed in lower resolution merger simulations (e.g., Barnes \& Hernquist 1996; Barnes 2002). In simulations with star formation, these large inflows result in a central starburst and more than $90 \%$ of the central gas distribution is converted into stars in less than $10^{8} \mathrm{yr}$. The peak star formation rates range from 30 to $>100 M_{\odot} \mathrm{yr}^{-1}$, comparable to those of luminous infrared galaxies and ultraluminous infrared galaxies (ULIRGs), suggesting that our modeling of star formation and the amount of cold gas present are realistic. The two SMBHs end up orbiting at the center of the remnant on eccentric orbits and form a close pair at a separation comparable to the adopted force resolution of $\sim 100 \mathrm{pc}$. Significantly higher mass and force resolution would be needed to follow the SMBHs at relative separations where they would form a binary system.

In unequal-mass mergers, the outcome depends sensitively on how the internal structure of the merging galaxies is modified by dissipation. In collisionless simulations, the tidal disruption of the satellite galaxy at about $6 \mathrm{kpc}$ from the center leaves its SMBH wandering at a distance that prohibits the formation of a close pair. This "naked" SMBH would contribute to a population of wandering SMBHs in galactic halos (Volonteri et al. 2003). At such a distance, the timescale for orbital decay predicted by dynamical friction would exceed a Hubble time. In simulations with cooling, the situation is completely reversed, as the gas inflow becomes particularly strong in the companion galaxy. We measured an inflow of about $3 M_{\odot} \mathrm{yr}^{-1}$ within approximately the central kiloparsec, compared with $<0.5 M_{\odot} \mathrm{yr}^{-1}$ for the larger galaxy. This striking difference in the gas inflows is attributed to a tidally induced strong stellar bar observed in the satellite galaxy approximately $1.5 \mathrm{Gyr}$ before the final passage. More than $50 \%$ of the gas is funneled into the center owing to gas shocking in the bar potential. At this stage, the companion galaxy has about $20 \%$ more gas within approximately its central kiloparsec relative to the larger galaxy. Dissipation enables the core of the infalling galaxy to survive complete tidal disruption by deepening its potential well, resulting in an SMBH pair separated by $\sim 100 \mathrm{pc}$ as in the equal-mass mergers.

The large gas inflows observed in the cooling and star formation simulations always produce a rotationally supported nuclear disk with a size in the range of $1-2 \mathrm{kpc}$ and a temperature set by our temperature floor $\left(T=2 \times 10^{4} \mathrm{~K}\right)$. In adiabatic simulations, the gas remains in a hot phase and forms a pressuresupported cloud of similar size. The reported nuclear disks are tilted by several to a few tens of degrees relative to the orbital plane of the galaxies. They have peak rotational velocities and masses in the range of $250-300 \mathrm{~km} \mathrm{~s}^{-1}$ and $\sim 10^{8}-10^{9} M_{\odot}$, respectively, and they are well resolved $\left(N \gtrsim 10^{4}\right)$. Nuclear disks of molecular gas of a few hundred parsecs to approximately a kiloparsec scale have been identified spectroscopically for several AGNs and ULIRGs (e.g., Davies et al. 2004), whose observed sizes, masses, and rotational velocities are comparable to those measured in our simulations. The nuclear disks reported here provide the reservoir of gas that fuels the central SMBHs.

\section{2. $M_{\mathrm{BH}^{-}} \sigma$ Relation in Binary Disk Galaxy Mergers}

For each remnant, we calculate line-of-sight aperture stellar velocity dispersions $\sigma$ within one effective radius $R_{e}$ (Gebhardt et al. 2000) and we use a range of viewing orientations, namely, inclinations $i=0^{\circ}, 60^{\circ}$, and $90^{\circ}$. For $i=60^{\circ}$ and $i=90^{\circ}$, we rotate the system about an axis perpendicular to the inclination axis through an angle $\phi$ set to $0^{\circ}, 45^{\circ}, 90^{\circ}$, and $135^{\circ}$. Thus, for each simulation we obtain nine different orientations. For each of these viewing angles, we measured the mass density profiles in circular apertures out to two radii $(8$ and $25 \mathrm{kpc})$ under two assumptions: (1) the system is a disk galaxy, and we decompose the mass density profile into a Sérsic bulge and an exponential disk; (2) the system is a pure spheroid, and we fit it with a pure Sérsic profile. This procedure ensures that the measurements of $R_{e}$, and therefore of $\sigma$, that we obtain are very robust.

In cases when the two SMBHs form a close pair, the $M_{\mathrm{BH}}$ assigned to each remnant is calculated by summing the masses of the two SMBHs and adding the gas mass contained within the resolution of our gasdynamical simulations. In doing this, we implicitly assume that any close pair will actually form an SMBH binary that will subsequently coalesce. Indeed, this is supported by the smaller scale simulations of Escala et al. (2004a, 2004b), who showed that SMBH pairs starting at a separation of few hundred parsecs in a gaseous background will typically merge within $\sim 10^{7}$ yr. When the SMBHs do not form a close pair, we only consider the mass of the SMBH located closer to the center of the remnant. Figure 2 shows the location of all merger remnants on the $M_{\mathrm{BH}^{-}}-\sigma$ plane together with real galaxy data and their best-fit correlation taken from Tremaine et al. (2002). We note that the initial galaxy models start very close to the mean correlation by construction. The 
error bars show the spread about the mean value of $\sigma$ and are smaller in the cooling and star formation simulations, likely because of the fact that these remnants are more spherical (Kazantzidis et al. 2004a). Mergers lead to an increase of the stellar velocity dispersion that is remarkably different depending on the physics included in the simulations. The largest increase occurs in simulations with radiative cooling, which exhibit the deepest potentials. When star formation is included, we measure velocity dispersions closer to that of the collisionless simulations, since the gas does not build a significant central mass concentration.

Figure 2 illustrates that collisionless mergers move remnants away from the mean $M_{\mathrm{BH}^{-}} \sigma$ relation. In adiabatic simulations, the galaxies have in principle enough gaseous fuel to remain close to the relation, but the gas is too hot to accrete onto the SMBHs. In mergers with radiative cooling, the total amount of nuclear gas is more than 1 order of magnitude larger than needed to keep the galaxies close to the relation. This should also be regarded as an upper limit on the gas mass that may accrete onto the SMBHs, since feedback processes ensuing once the AGN becomes active will regulate the inflow and allow the accretion of only a fraction of this gas, thus limiting the SMBH growth (Silk \& Rees 1998). If AGN feedback is strong enough to suppress cooling, a hot gas phase similar to that found in our adiabatic simulations is expected to form. Thus, the results from cooling and adiabatic simulations likely provide upper and lower bounds to the true physical behavior of the resulting remnants. Note that the point corresponding to the $4: 1$ merger is the highest with respect to the best-fit correlation in Figure 2. This is the product of the particularly strong gas inflow occurring in the satellite galaxy just before the merger is completed. Overall, a higher fraction of the available gas is driven toward the center relative to the equal-mass galaxy mergers. When star formation is included, the amount of cold gas in the nuclear disks is reduced by more than $90 \%$ and the merger remnants move nearly parallel to the relation. This suggests that star formation during mergers is a key ingredient for maintaining the tightness of the $M_{\mathrm{BH}^{-}} \sigma$ relation.

\section{DISCUSSION AND CONCLUSIONS}

Gaseous dissipation influences considerably the outcome of binary mergers of disk-dominated galaxies containing SMBHs. Most importantly, it controls the $\mathrm{SMBH}$ pairing process in unequal-mass mergers by modifying the central structure of the companion galaxy, enabling it to survive complete tidal disruption. Dissipationless, unequal-mass mergers are also expected to produce close SMBH pairs when the satellite galaxy is dominated by a dense stellar central component, as in nucleated dwarf elliptical (e.g., M32) and S0 galaxies. Our results suggest that semianalytic models of hierarchical SMBH growth that neglect the effect of dissipation on the orbital evolution of SMBHs underestimate their pairing efficiency and subsequent coalescence. This consequently leads to overestimating the number of wandering SMBHs in Milky Way-sized galaxies (Volonteri et al. 2003). The higher pairing efficiency of $\sim 10^{6} M_{\odot}$ SMBHs reported here has important implications for the probability of observing coalescence events with space-based gravitational waves experiments such as the Laser Interferometer Space Antenna (Sesana et al. 2004).

Gaseous dissipation also determines how merging galaxies constructed to satisfy the $M_{\mathrm{BH}^{-}} \sigma$ relation move relative to their initial location on the $M_{\mathrm{BH}^{-}}-\sigma$ plane. The collisionless simulations reveal that when galaxies become gas-poor, which is likely at low $z$, their merger remnants will tend to move away from the mean relation. This suggests that gas-poor mergers act as a possible source of scatter in the mean $M_{\mathrm{BH}^{-}} \sigma$ relation. The natural scaling between the amount of cold nuclear gas and the increase in the central stellar velocity dispersion explains why merger remnants in simulations with radiative cooling move above the mean relation. Star formation serves to move merger remnants nearly parallel to the relation. The fact that unequalmass mergers appear effective at building a reservoir of gas for SMBH accretion is noteworthy, since in hierarchical structure formation models they are significantly more frequent than equal-mass ones. Finally, the nuclear disks found in this study show significant spiral patterns, which are sustained by the selfgravity of the disks (Mayer et al. 2004). We measure typical inflows in the range $10^{-2}$ to $10^{-3} M_{\odot} \mathrm{yr}^{-1}$ within $1 \mathrm{kpc}$. Such inflows are expected to feed the SMBHs and bridge the gap between the large-scale flows and the viscous accretion taking place once the gas has reached the AGN accretion disk at subparsec scales (e.g., Heller \& Shlosman 1994). Future simulations will be used to explore the feasibility of such mechanisms.

S. K. would like to thank Aaron Dutton, Andrey Kravtsov, and David Merritt for useful discussions. P. M. acknowledges support by NASA grants NAG5-11513 and NNG04GK85G and by NSF grant AST-0205738. The numerical simulations were performed on the zBox supercomputer at the University of Zürich and on the Intel cluster at the Cineca Supercomputing Center in Bologna (Italy).

\section{REFERENCES}

Barnes, J. E. 2002, MNRAS, 333, 481

Barnes, J. E., \& Hernquist, L. 1996, ApJ, 471, 115

Blumenthal, G. R., Faber, S. M., Flores, R., \& Primack, J. R. 1986, ApJ, 301, 27

Davies, R. I., Tacconi, L. J., \& Genzel, R. 2004, ApJ, 602, 148

Escala, A., Larson, R. B., Coppi, P. S., \& Mardones, D. 2004a, ApJ, 607, 765 . 2004b, ApJ, submitted (astro-ph/0406304)

Ferrarese, L., \& Merritt, D. 2000, ApJ, 539, L9

Gebhardt, K., et al. 2000, ApJ, 539, L13

Governato, F., Colpi, M., \& Maraschi, L. 1994, MNRAS, 271, 317

Heller, C. H., \& Shlosman, I. 1994, ApJ, 424, 84

Hernquist, L. 1990, ApJ, 356, 359 1993, ApJS, 86, 389

Katz, N. 1992, ApJ, 391, 502

Kazantzidis, S., Kravtsov, A. V., Zentner, A. R., Allgood, B., Nagai, D., \& Moore, B. 2004a, ApJ, 611, L73
Kazantzidis, S., Magorrian, J., \& Moore, B. 2004b, ApJ, 601, 37

Klypin, A., Zhao, H., \& Somerville, R. S. 2002, ApJ, 573, 597

Komossa, S., Burwitz, V., Hasinger, G., Predehl, P., Kaastra, J. S., \& Ikebe, Y. 2003, ApJ, 582, L15

Kormendy, J., \& Richstone, D. 1995, ARA\&A, 33, 581

Makino, J., \& Ebisuzaki, T. 1996, ApJ, 465, 527

Mayer, L., Quinn, T., Wadsley, J., \& Stadel, J. 2004, ApJ, 609, 1045

Milosavljević, M., \& Merritt, D. 2001, ApJ, 563, 34

Mo, H. J., Mao, S., \& White, S. D. M. 1998, MNRAS, 295, 319

Navarro, J. F., Frenk, C. S., \& White, S. D. M. 1996, ApJ, 462, 563

Sesana, A., Haardt, F., Madau, P., \& Volonteri, M. 2004, ApJ, 611, 623

Silk, J., \& Rees, M. J. 1998, A\&A, 331, L1

Taffoni, G., Mayer, L., Colpi, M., \& Governato, F. 2003, MNRAS, 341, 434

Tremaine, S., et al. 2002, ApJ, 574, 740

Volonteri, M., Haardt, F., \& Madau, P. 2003, ApJ, 582, 559

Wadsley, J. W., Stadel, J., \& Quinn, T. 2004, NewA, 9, 137 